\title{
ON IRREDUCIBLE POLYNOMIALS IN SEVERAL VARI- ABLES WHICH BECOME REDUCIBLE WHEN THE VARIABLES ARE REPLACED BY POWERS OF THEMSELVES* \\ BY \\ ELI GOURIN
}

Let $Q\left(x_{1}, \cdots, x_{s}\right)$ be a polynomial in $x_{1}, \cdots, x_{s}$, irreducible in the field of all constants. There exist, in certain cases, integers $t_{1}, \cdots, t_{s}$ such that the polynomial $Q\left(x_{1}^{t_{1}}, \cdots, x_{s}{ }^{t_{0}}\right)$ is reducible.

The determination of the integers $t$ for which reducibility occurs is a problem which arose in an investigation of J. F. Ritt on the factorization of exponential forms. $\dagger$ The case of real interest is that in which $Q$ has at least three terms. We shall, in this introduction, limit ourselves to the discussion of the results for this case. The relatively simple case of two terms is treated in Part III.

For a fairly general, but not perfectly general, type of polynomial $Q$, consisting of more than two terms, Ritt proved that the sets $t$ break up into a finite number of classes, the sets of any one class being, from a certain point of view, equivalent.

In the present paper, we obtain information relative to the sets $t$ which, in certain respects, is final. Our results are embodied in the following theorems, in which each $t_{i}$ is understood to be a positive integer.

Theorem I. Let $Q\left(x_{1}, \cdots, x_{s}\right)$ be an absolutely irreducible polynomial, consisting of more than two terms. Suppose that at least one set $t_{1}, \cdots, t_{s}$ exists such that $Q\left(x_{1}^{t_{1}}, \cdots, x_{s}^{t^{t}}\right)$ is reducible. Then there exists one and only one finite aggregate of sets

$$
t_{11}, \cdots, t_{1 s} ; \cdots ; t_{n 1}, \cdots, t_{n s}
$$

which fulfill the following conditions:

(1) For every $i, Q\left(x_{1}^{t^{i_{11}}}, \cdots, x_{s}{ }^{\left.t_{i s}\right)}\right.$ is reducible.

(2) If $Q\left(x_{1}{ }^{t_{1}}, \cdots, x_{s}{ }^{t_{0}}\right)$ is reducible, there exists one and only one set $t_{j 1}, \cdots, t_{j s}$ such that each $t_{k}$ is an integral multiple of $t_{j k}$ and such that if $t_{k}=a_{k} t_{j k}$ $(k=1, \cdots, s)$, then the irreducible factors of $Q\left(x_{1}^{t_{1}}, \cdots, x_{s} t_{s}\right)$ are found by replacing each $x_{k}$ by $x_{k}{ }^{a_{k}}$ in the irreducible factors of $Q\left(x_{1}{ }^{t_{11}}, \cdots, x_{s}{ }^{t_{s}}\right) . \ddagger$

* Presented to the Society, February 23, 1929; received by the editors in February, 1930.

$\dagger$ A factorization theory for functions $\sum_{i=1}^{n} a_{i} e^{\alpha_{i x}}$, these Transactions, vol. 29 (1927), pp. 584-596.

$\ddagger$ That is, if in any irreducible factor of $Q\left(x_{1}{ }^{t} j 1, \cdots, x_{s}{ }^{t_{j}}\right)$ each $x_{k}$ is replaced by $x_{k}{ }^{a_{k}}$ the resulting polynomial will be irreducible. 
We shall call each of the $n$ sets of $(\alpha)$ a basic set.* With respect to such sets we obtain

Theorem II. Let $M=\max \left(M_{1}, \cdots, M_{s}\right)$, where $M_{i}$ is the degree of $Q\left(x_{1}, \cdots, x_{s}\right)$ in $x_{i}$. For any element $t_{i j}$ of any of the basic sets of $Q$ we have $t_{i j} \leqq M^{2}$.

Furthermore, the bound $M^{2}$ is the smallest possible bound. We construct polynomials $Q$ for which this bound is actually attained.

The above results are stronger than those of Ritt in the following respects. Ritt dęals with a type of polynomial $Q$ which he calls primary (see $\$ 1$ ), and supposes that one of the terms of $Q$ is unity. In our case, $Q$ is any polynomial with more than two terms. But the chief advance of the present paper is the determination of the best upper bound, $M^{2}$, for the elements of the basic sets. The bound given by Ritt for the case with which he deals is $\delta^{*+4}$, where $\delta$ is the degree of $Q$. It may be remarked that our method of proof is essentially simpler than that of Ritt. His complicated first lemma is eliminated entirely.

In Part II, we define a set $t_{1}, \cdots, t_{s}$ as minimal if $Q\left(x_{1}{ }^{t_{1}}, \cdots, x_{s}{ }^{t}\right)$ is reducible, but if no $Q\left(x_{1}^{r_{1}}, \cdots, x_{s}^{r_{i}}\right)$ with each $\tau_{i}$ a submultiple of $t_{i}$, at least one $\tau_{i}$ a proper submultiple of its $t_{i}$, is reducible.

We prove, for a polynomial $Q$ consisting of more than two terms,

THEOREM III. Those elements of a minimal set which are distinct from unity are equal to each other, and their common value is a prime number which does not exceed the greatest prime less than $M^{2}$.

Furthermore we construct polynomials for which the upper bound given in Theorem III is actually attained.

\section{BASIC SETS}

1. We shall say that two polynomials, neither identically zero, are equivalent if their ratio is a constant.

Let it be understood that no term of the polynomial $Q\left(x_{1}, \cdots, x_{s}\right)$ has a zero coefficient and that each $x$ is present in some term of $Q$ with an exponent greater than 0 . Following Ritt, we shall say that $Q$ is primary in $x_{i}$ if the highest common factor of the exponents of $x_{i}$ in all the terms of $Q$ is unity. If $Q$ is primary in each of its variables, we say, simply, that $Q$ is primary.

Let $t_{1}, \cdots, t_{\mathrm{s}}$ be positive integers. Consider the group $G$ of substitutions which replace the variables $x_{1}, \cdots, x_{s}$ by $\epsilon_{1}{ }^{k_{1}} x_{1}, \cdots, \epsilon_{s}{ }^{k_{s}} x_{s}$, respectively,

* Assuming the existence of these sets, it is easy to conclude that no infinite system of distinct sets satisfying the conditions (1) and (2) of the theorem can exist. See also $\$ 6$. 
where $\epsilon_{i}$ is a primitive $t_{i}$ th root of unity and $k_{i}$ is an arbitrary integer. $G$ is of order $t_{1} t_{2} \cdots t_{s}$.

The $t_{1} t_{2} \cdots t_{\text {. }}$ transforms of a given polynomial by means of $G$ can be grouped into classes of equivalent polynomials. A set of transforms obtained by choosing one polynomial from each such class will be referred to as a complete set of transforms.

2. We shall prove the following lemma:

LEMMA I. If $Q\left(x_{1}, \cdots, x_{s}\right)$ is irreducible and $Q^{(t)}=Q\left(x_{1}^{t_{1}}, \cdots, x_{s}^{t^{c}}\right)$ is reducible, then the irreducible factors $Q_{1}, \cdots, Q_{N}$ of $Q^{(t)}$ form a complete set of transforms obtained from any one of them.

Because $Q$ is irreducible, no monomial can be a factor of $Q$. Hence $Q^{(t)}$ can have no monomial factor, either. Thus $Q_{1}$ contains at least two terms.

It is obvious that every substitution of $G$ leaves $Q^{(t)}$ invariant. Consequently, any polynomial obtained from $Q_{1}$ by means of $G$ is, like $Q_{1}$, an irreducible factor of $Q^{(t)}$.

Let $Q_{1}, \cdots, Q_{l}$ be a complete set of transforms obtained from $Q_{1}$. The product $P=Q_{1} \cdots Q_{l}$, for any substitution of $G$, goes over into a polynomial equivalent to $P$. Furthermore, as the $Q_{i}$ 's are irreducible, and relatively prime, $P$ must be a factor of $Q^{(t)}$. Accordingly, let

$$
Q^{(t)}=P \cdot R \text {. }
$$

We shall prove that $R$ is a constant.

Consider any single variable, say $x_{1}$. Not every term of $P$ can contain $x_{1}$, else $P$, and therefore $Q^{(t)}$, would have a monomial factor. Hence the substitution of $G$ which replaces $x_{1}$ by $\epsilon_{1} x_{1}$ and leaves all other variables unchanged must leave $P$ invariant. Consequently, $P$ is a rational function of $x_{1}{ }^{t_{1}}$ and, similarly, of $x_{i}{ }^{i}$, for every $i$. If, in (1), $R$ were not a constant, it would certainly be, as the quotient of $Q^{(t)}$ by $P$, a rational and, consequently, an integral rational function of every $x_{i}{ }^{t_{i}}$. Thus $R$ must be a constant, else $Q$ would be reducible.

We may assume that $R$ is unity. Where this is not so at the start, it can be brought about by multiplying $Q_{1}$ by $1 / R^{1 / N}$.

Our lemma is thus proved.

Corollary. Each $Q_{i}$ in the identity $Q^{(t)}=Q_{1} \cdots Q_{N}$ involves every variable $x_{j}$.

3. We assume now that the irreducible factor $Q_{1}$ of $Q^{(t)}$ is primary and 
contains at least three terms. Because $Q_{1}$ is irreducible, it must contain at east one term independent of $x_{1}$. Suppose, then, that

(2)

$$
\begin{aligned}
& Q_{1}=A_{1} x_{1}^{\alpha_{1}} x_{2}^{\alpha_{2}} \cdots x_{8}^{\alpha_{t}}+A_{2} x_{1}^{\beta_{1}} x_{2}{ }^{\beta_{2}} \cdots x_{8}^{\beta_{s}}
\end{aligned}
$$



where the $A$ 's are constants and the last term does not contain $x_{1}$.

From (2) we find

$$
Q_{1}=x_{2}{ }^{{ }^{2}} \cdots x_{8}{ }^{{ }^{2}} U
$$

where

$$
\begin{aligned}
U=A_{1} x_{1}{ }^{\alpha_{1}} x_{2}{ }^{\alpha_{2}-\nu_{2}} \cdots x_{s}{ }^{\alpha_{s}-\nu_{s}}+A_{2} x_{1}^{\beta_{1}} x_{2}{ }^{\beta_{2}-\nu_{2}} \cdots x_{8}^{\beta_{s}-\nu_{s}} \\
+\cdots+A_{\mu} x_{1}^{{ }^{\mu_{1}}} x_{2}{ }^{\mu_{2}-\nu_{2}} \cdots x_{8}{ }^{\mu_{s}-\nu_{s}}+A_{v}
\end{aligned}
$$

LEMma II. If $Q_{1}$ contains at least three terms and is primary, then, for at least one variable $x_{i}(i \neq 1)$ the exponents $\alpha_{1}, \beta_{1}, \cdots, \mu_{1}$ in $U$ will not be proportional to the corresponding exponents $\alpha_{i}-\nu_{i}, \beta_{i}-\nu_{i}, \cdots, \mu_{i}-\nu_{i}$.

Suppose that this is not true. Then, since the exponents of $x_{1}$ in the various terms of $U$ are all non-negative integers, the exponents of any other variable $x_{j}$ must be either all non-positive or all non-negative. Let $D_{i}$ be the greatest common factor of the exponents of $x_{i}$ in $U$ (if they are non-positive, we shall understand by $D_{j}$ their common negative divisor of maximum absolute value).

The exponents of $x_{j}$ in $U$ will, accordingly, be of the form $a_{1 j} D_{j}, \cdots$, $a_{\mu j} D_{j}$, where the $a^{\prime}$ s are relatively prime non-negative integers. When $j=1, D=1$, because $U$ has the same exponents for $x_{1}$ as $Q_{1}$. From this fact and the assumed proportionality of the exponents it follows immediately that, for any $j$, the set of integers $a_{1 j}, \cdots, a_{\mu j}$ is identical with the set $\alpha_{1}, \cdots, \mu_{1}$. Consequently, $U$ is a polynomial in the product $z=x_{1} x_{2}{ }^{D_{2}} \cdots x_{s}{ }^{D_{9}}$. Then

$$
U=C_{0} z^{n}+C_{1} z^{n-1}+\cdots+C_{n+1},
$$

with constant $C$ 's and $n>1$.

If $z_{1}, \cdots, z_{n}$ are the zeros of this latter polynomial, we find, as a consequence from (3) and (5),

$$
Q_{1}=C_{0} x_{2}{ }^{v_{2}} \cdots x_{8}{ }^{{ }_{s}}\left(z-z_{1}\right) \cdots\left(z-z_{n}\right) .
$$

The product $z=x_{1} x_{2} D_{2} \cdots x_{s}^{D} \cdot$ must involve negative powers; otherwise, by (6), $Q$ would be reducible. Fixing our ideas, let us assume that $D_{2}$, $D_{3}, \cdots, D_{k}$, but no other $D$ 's, are negative. Because the exponents of $Q_{1}$ are non-negative, we conclude from (6) that in the expressions 


$$
d_{2}=\nu_{2}+D_{2} n, d_{3}=\nu_{3}+D_{3} n, \cdots, d_{k}=\nu_{k}+D_{k} n
$$

the $d$ 's are non-negative integers.

From (6) and (7) we have that

$$
\begin{aligned}
& Q_{1}=C_{0} x_{2}{ }^{d_{2}} \cdots x_{k}{ }^{d_{k}} x_{k+1}{ }^{v_{k+1}} \cdots x_{\mathrm{s}}{ }^{p_{s}}\left[x_{2}^{-D_{2}}\right.
\end{aligned}
$$

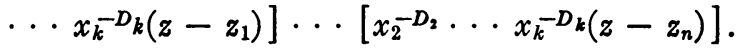

Each of the factors $x_{2}^{-D_{2}} \cdots x_{k}^{-D_{k}\left(z-z_{i}\right)}$ is a binomial in which the variables involved have positive exponents. Because $n$ is greater than unity, there are at least two such factors. This result is, however, an absurdity, since $Q_{1}$ is irreducible.

Thus in $U$, for some $x_{i}$, the exponents are not proportional to those of $x_{1}$.

4. We shall prove the following lemma:

Lemma III. If an irreducible factor $Q_{1}$ of $Q^{(t)}=Q\left(x_{1}{ }^{t 1}, \cdots, x_{s}^{t} \cdot\right)$ consists of at least three terms, and is primary, then each $t_{j}$, where $j$ is among the numbers $1,2, \cdots, s$, satisfies the relation $t_{j} \leqq M^{2}$.

It follows from Lemma I that, if $Q^{(t)}=Q_{1} \cdots Q_{N}$, and $Q_{1}$ consists of more than two terms and is primary, then each of the $Q_{i}$ 's contains, likewise, more than two terms and is primary.

We conclude further from Lemma II, and from the expression of $Q_{1}$ as given in (2), that there exists a subscript $i$ such that at least two numbers in the set $\alpha_{1}, \beta_{1}, \cdots, \mu_{1}$ are not proportional to two corresponding numbers in the set $\alpha_{i}-\nu_{i}, \beta_{i}-\nu_{i}, \cdots, \mu_{i}-\nu_{i}$. Changing, if necessary, the subscripts of the variables as well as the notation of the exponents in (2), we may assume that the numbers $\alpha_{1}, \beta_{1}$ are not proportional to $\alpha_{2}-\nu_{2}, \beta_{2}-\nu_{2}$ and that the determinant $\alpha_{1}\left(\beta_{2}-\nu_{2}\right)-\beta_{1}\left(\alpha_{2}-\nu_{2}\right)$ is positive.

Let $n_{1}$ be the degree of $Q_{1}$ in $x_{1}$ and $n_{2}$ the degree in $x_{2}$. Let $m_{1}$ and $m_{2}$ be, respectively, the degrees of $Q$ in the same variables. Because $Q_{1}$ is primary, there will be no two equivalent polynomials among the $t_{1}$ polynomials $Q_{1}\left(\epsilon_{1}{ }^{{ }_{1}} x_{1}, x_{2}, \cdots, x_{s}\right)\left(k_{1}=0,1, \cdots, t_{1}-1\right)$.

Suppose, indeed, that, for distinct values $p$ and $q$ of $k_{1}$, the corresponding polynomials $Q_{1 p}$ and $Q_{1 q}$ satisfy a relation

$$
Q_{1 p}=c Q_{1 q},
$$

where $c$ is a constant. It is easy to see from (2) that $c$ must be unity and that

or

$$
\begin{aligned}
& \epsilon_{1}{ }^{p \alpha_{1}}=\epsilon_{1}{ }^{\alpha_{1}}, \cdots, \epsilon_{1}{ }^{p \mu_{1}}=\epsilon_{1}{ }^{q \mu_{1}}, \\
& \frac{\alpha_{1}(p-q)}{t_{1}}=I_{\alpha}, \cdots, \frac{\mu_{1}(p-q)}{t_{1}}=I_{\mu},
\end{aligned}
$$


where the I's are integers. Because $\alpha_{1}, \cdots, \mu_{1}$ are relatively prime, $p-q$ must be divisible by $t_{1}$, which implies that $p=q$.

It follows therefore from Lemma I that $N$ is at least equal to $t_{1}$. By comparing the degrees in $x_{1}$ of both sides of the identity

$$
Q^{(t)}=Q_{1} \cdot Q_{2} \cdots Q_{N}
$$

we find that

$$
t_{1} n_{1} \leqq t_{1} m_{1}, \quad \text { or } n_{1} \leqq m_{1} .
$$

Similarly, we find that

$$
t_{2} n_{2} \leqq t_{2} m_{2}, \quad \text { or } n_{2} \leqq m_{2} .
$$

Let us now denote by $Q_{u v}$ any of the polynomials which are obtained from $Q_{1}$ by placing $\epsilon_{1}{ }^{u} x_{1}$ for $x_{1}$ and $\epsilon_{2}{ }^{0} x_{2}$ for $x_{2}$, where $u$ is among the numbers $0,1, \cdots, t_{1}-1$ and $v$ among the numbers $0,1, \cdots, t_{2}-1$.

These $t_{1} t_{2}$ polynomials may form several sets of equivalent polynomials. Suppose, therefore, that, for two pairs of integers $\left(u_{1}, v_{1}\right)$ and $\left(u_{2}, v_{2}\right)$, the corresponding polynomials $Q_{u_{1} v_{1}}$ and $Q_{u_{2} v_{2}}$ are equivalent. It follows then from (2) by considering the first two and the last terms, that the following relations must be satisfied:

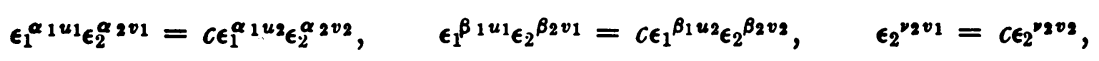

where $c$ is a constant. This implies that

$$
\begin{aligned}
\frac{\alpha_{1}\left(u_{2}-u_{1}\right)}{t_{1}}+\frac{\alpha_{2}\left(v_{2}-v_{1}\right)}{t_{2}} & =I_{1}+e, \\
\frac{\beta_{1}\left(u_{2}-u_{1}\right)}{t_{1}}+\frac{\beta_{2}\left(v_{2}-v_{1}\right)}{t_{2}} & =I_{2}+e, \\
\frac{\nu_{2}\left(v_{2}-v_{1}\right)}{t_{2}} & =I_{3}+e,
\end{aligned}
$$

where the $I$ 's are integers and $e$ is a rational fraction.

Subtracting (14) from (12) and (13), we find that

$$
\begin{aligned}
& \frac{\alpha_{1}\left(u_{2}-u_{1}\right)}{t_{1}}+\frac{\left(\alpha_{2}-\nu_{2}\right)\left(v_{2}-v_{1}\right)}{t_{2}}=I_{4}, \\
& \frac{\beta_{1}\left(u_{2}-u_{1}\right)}{t_{1}}+\frac{\left(\beta_{2}-\nu_{2}\right)\left(v_{2}-v_{1}\right)}{t_{2}}=I_{5},
\end{aligned}
$$


and, solving for $\left(u_{2}-u_{1}\right) / t_{1}$ and $\left(v_{2}-v_{1}\right) / t_{2}$, we have

$$
\begin{aligned}
& \frac{\left(u_{2}-u_{1}\right) \Delta}{t_{1}}=I_{6}, \\
& \frac{\left(v_{2}-v_{1}\right) \Delta}{t_{2}}=I_{7},
\end{aligned}
$$

where $I_{4}, \cdots, I_{7}$ are integers and $\Delta$ is the determinant $\alpha_{1}\left(\beta_{2}-\nu_{2}\right)$ $-\beta_{1}\left(\alpha_{2}-\nu_{2}\right)$.

Let $d_{i}(i=1,2)$ be the highest common factor of $\Delta$ and $t_{i}(i=1,2)$. Then

$$
\Delta=d_{1} \delta_{1}=d_{2} \delta_{2}, \quad t_{1}=d_{1} \tau_{1}, \quad t_{2}=d_{2} \tau_{2},
$$

where $\delta_{1}, \delta_{2}, \tau_{1}, \tau_{2}$ are positive integers.

Substituting these expressions in (15) and (16) we find that

$$
\begin{aligned}
& \frac{u_{2}-u_{1}}{\tau_{2}} \delta_{1}=I_{6}, \\
& \frac{v_{2}-v_{1}}{\tau_{2}} \delta_{2}=I_{7} .
\end{aligned}
$$

Since the pairs $\left(\delta_{1}, \tau_{1}\right)$ and $\left(\delta_{2}, \tau_{2}\right)$ are relatively prime, the relations (17) and (18) can be satisfied only if $\left(u_{2}-u_{1}\right)$ is divisible by $\tau_{1}$ and $\left(v_{2}-v_{1}\right)$ by $\tau_{2}$.

It follows immediately that these relations will surely not be satisfied if $u_{1}$ and $u_{2}$ are both among the numbers $0,1, \cdots, t_{1}-1$, and $v_{1}$ and $v_{2}$ both among the numbers $0,1, \cdots, \tau_{2}-1$. Thus we can obtain from $Q_{1}$, by means of the substitutions of $G$, at least $t_{1} \tau_{2}$ polynomials no two of which are equivalent. This implies that $N \geqq t_{1} \tau_{2}$. Comparing the degrees in $x_{2}$ of both sides of the identity (9), we find that

$$
t_{1} \tau_{2} n_{2} \leqq t_{2} m_{2},
$$

or, since $\tau_{2}=t_{2} / d_{2}$,

$$
t_{1} \leqq \frac{m_{2} d_{2}}{n_{2}} \leqq \frac{m_{2} \Delta}{n_{2}} .
$$

Now $\Delta$ stands for the expression $\alpha_{1}\left(\beta_{2}-\nu_{2}\right)-\beta_{1}\left(\alpha_{2}-\nu_{2}\right)$, which is positive. If, therefore, both $\beta_{2}-\nu_{2}$ and $\alpha_{2}-\nu_{2}$ are non-negative, then $\Delta \leqq \alpha_{1}\left(\beta_{2}-\nu_{2}\right)$ $\leqq \alpha_{1} n_{2}$. If they are both non-positive, then $\Delta \leqq \beta_{1}\left(\nu_{2}-\alpha_{2}\right) \leqq \beta_{1} n_{2}$. Finally, if $\beta_{2}-\nu_{2}$ is positive and $\alpha_{2}-\nu_{2}$ is negative, then

$\Delta=\alpha_{1}\left(\beta_{2}-\nu_{2}\right)+\beta_{1}\left(\nu_{2}-\alpha_{2}\right) \leqq n_{1}\left(\beta_{2}-\nu_{2}\right)+n_{1}\left(\nu_{2}-\alpha_{2}\right) \leqq n_{1}\left(\beta_{2}-\alpha_{2}\right) \leqq n_{1} n_{2}$.

We find, thus, that in all cases $\Delta \leqq n_{1} n_{2}$. Hence, it follows from (20) that 


$$
t_{1} \leqq m_{2} n_{1}
$$

and, from (10), that

$$
t_{1} \leqq m_{2} m_{1} \leqq M^{2}
$$

This result we obtained by considering a particular variable $x_{1}$. We can apply the same reasoning to any other variable $x_{i}$ and prove that, in general,

$$
t_{i} \leqq M^{2} \text {. }
$$

Lemma III is proved.

5. We assume, next, that the irreducible polynomial $Q\left(x_{1}, \cdots, x_{8}\right)$ consists of at least three terms and prove

LEMMA IV. If $Q$ consists of at least three terms and if the irreducible factor $Q_{1}$ of $Q^{(t)}$ is primary, then $Q_{1}$ consists, likewise, of at least three terms.

Suppose that $Q_{1}$ contains just two terms.

Because $Q_{1}$ is irreducible, a given variable $x_{i}$ can be present only in one of the two terms. Further, since $Q_{1}$ is primary, it can involve only first powers of the variables. Let us assume, therefore, since we are free to interchange the subscripts, that

$$
Q_{1}=a x_{1} x_{2} \cdots x_{k}+b x_{k+1} \cdots x_{s},
$$

where $a$ and $b$ are constants.

We denote by $q$ the ratio $x_{k+1} \cdots x_{s} /\left(x_{1} x_{2} \cdots x_{k}\right)$, so that $Q_{1}=a x_{1} \cdots x_{k}$ $\left(1+c_{1} q\right)$, where $c_{1}=b / a$.

It follows from Lemma I that, if $Q^{(t)}=Q_{1} Q_{2} \cdots Q_{N}$, then each $Q_{i}$ will be of the form $Q_{i}=d_{i} a x_{1} \cdots x_{k}\left(1+c_{i} q\right)$, where $d_{i}$ and $c_{i}$ are constants and $d_{1}=1$.

We find, in this way, the following expression for $Q^{(t)}$ :

$$
Q^{(t)}=D a^{N}\left(x_{1} x_{2} \cdots x_{k}\right)^{N}\left(1+B_{1} q^{\alpha_{1}}+B_{2} q^{\alpha_{2}}+\cdots+B_{n} q^{N}\right),
$$

where $D=d_{1} d_{2} \cdots d_{N}$ and the last factor is the product of the $N$ binomials $\left(1+c_{i} q\right)$.

Since $Q^{(t)}$ does not change when $x_{i}(i=1, \cdots, k)$ is replaced by $\epsilon_{i} x_{i}$, it is easy to see that $N$ and each $\alpha_{j}$ must be divisible by $t_{i}$.

Similarly we conclude that $N$ and each of the $\alpha$ 's must be divisible by $t_{j}$, where $j$ is among the numbers $k+1, \cdots, s$. Consequently, $N=\nu T$ and $\alpha_{j}=\gamma_{j} T$, where $T$ is the least common multiple of the numbers $t_{1}, \cdots, t_{s}$, and $\nu$ and $\gamma_{j}$ are positive integers. Denoting $q^{T}$ by $q_{1}$, we find from (25)

$$
Q^{(t)}=D a^{N}\left(x_{1} \cdots x_{k}\right)^{\nu T}\left(1+B_{1} q_{1}^{\gamma_{1}}+B_{2} q_{1}^{\gamma_{2}}+\cdots+B_{n} q_{1}^{\nu}\right) .
$$


The exponent $\nu$ is greater than unity. Otherwise $Q^{(t)}$ and, consequently, $Q$ would contain only two terms. Then, the last factor of $Q^{(t)}$ in (26), as a polynomial in one variable of degree higher than unity, is reducible. Let $e_{1}, \cdots, e_{\nu}$ be the zeros of this polynomial.

We find from (26), substituting for $q_{1}$ its expression in the $x$ 's,

$$
\begin{array}{r}
Q^{(t)}=B_{n} D a^{N}\left[\left(x_{k+1} \cdots x_{\diamond}\right)^{T}-e_{1}\left(x_{1} \cdots x_{k}\right)^{T}\right] \\
\cdots\left[\left(x_{k+1} \cdots x_{\diamond}\right)^{T}-e_{v}\left(x_{1} \cdots x_{k}\right)^{r}\right],
\end{array}
$$

which contradicts the assumption that $Q$ is irreducible. Hence $Q_{1}$, and, consequently, each $Q_{i}$, consists of at least three terms.

6. An immediate consequence of the last two lemmas is the following result: Given an irreducible polynomial $Q\left(x_{1}, \cdots, x_{s}\right)$ consisting of more than two terms, there can exist only a finite number of sets

$$
t_{11}, \cdots, t_{18} ; \cdots ; t_{n 1}, \cdots, t_{n s}
$$

such that the irreducible factors of $Q\left(x_{1}{ }^{i_{i 1}}, \cdots, x_{s}{ }^{t_{i s}}\right)(i=1, \cdots, n)$ are primary.

We assume that all sets of such nature are present among the $n$ sets of $(\beta)$ and, furthermore, that no two sets of $(\beta)$ are identical.

We shall prove that if $Q$ is primary, then the sets $(\beta)$ are the basic sets of $Q$ referred to in the introduction. Let, indeed, $t_{1}, \cdots, t_{s}$ be a set of exponents for which $Q^{(t)}$ is reducible, and let $P\left(x_{1}, \cdots, x_{s}\right)$ be one of the irreducible factors of $Q^{(t)}$. If $\alpha_{i}$ is the greatest common factor of the exponents of $x_{i}$ in $P$, so that

$$
P\left(x_{1}, \cdots, x_{s}\right)=Q_{1}\left(x_{1}^{\alpha_{1}}, \cdots, x_{s}^{\alpha_{s}}\right)
$$

where $Q\left(x_{1}, \cdots, x_{s}\right)$ is primary, we conclude from Lemma I that

$$
Q^{(t)}=Q_{1}\left(x_{1} \alpha_{1}, \cdots, x_{8} \alpha_{s}\right) \cdots Q_{N}\left(x_{1}^{\alpha_{1}}, \cdots, x_{8}^{\alpha} s\right) .
$$

This implies that $Q^{(t)}$ is an integral rational function in $x_{j}{ }^{\alpha_{i}}(j=1, \cdots, s)$. Because $Q$ is primary, each $t_{j}$ must be divisible by the corresponding $\alpha_{j}$. If, therefore, $t_{j}=\alpha_{j} \tau_{j}$, we find from (28) that

$$
Q\left(x_{1}^{{ }^{\tau_{1}}}, \cdots, x_{s}{ }^{\tau_{s}}\right)=Q_{1}\left(x_{1}, \cdots, x_{s}\right) \cdots Q_{N}\left(x_{1}, \cdots, x_{s}\right)
$$

where the $Q_{i}$ 's are primary.

Consequently, the set $\tau_{1}, \cdots, \tau_{s}$ must be one of the sets $(\beta)$.

Hence, given any set of $t$ 's for which $Q^{(t)}$ is reducible, there always exists, among the sets $(\beta)$, one and only one set, say $t_{k 1}, \cdots, t_{k s}$, such that, for every $i, t_{i}=\alpha_{i} t_{k i}$ and such that the irreducible factors of $Q^{(t)}$ are obtained by replacing in the irreducible factors of $Q\left(x_{1}{ }^{t_{k 1}}, \cdots, x_{s}{ }^{t_{k}}\right)$ each $x_{i}$ by $x_{i}{ }^{\alpha_{i}}$. 
Furthermore, any other aggregate $(\gamma)$ of sets having the two properties just stated must be identical with the aggregate $(\beta)$. We shall prove, first, that any set of $(\beta)$ belongs to $(\gamma)$. Otherwise, we must conclude that there exists in $(\beta)$ a set, say $t_{i 1}, \cdots, t_{i s}$, whose elements are integral multiples of the corresponding elements of some set of $(\gamma)$, one element, at least, a proper multiple. Let $\tau_{1}, \cdots, \tau_{s}$ be the elements of this latter set. Each $\tau_{j}$ is, in its turn, a multiple of the corresponding element in some set, say $t_{k 1}, \cdots, t_{k e}$, of $(\beta)$, distinct from the set $t_{i 1}, \cdots, t_{i s}$. The elements of the two chosen sets of $(\beta)$ satisfy, therefore, relations of the form $t_{i j}=a_{j} t_{k j}$, at least one of the $a$ 's being greater than unity. Moreover, the irreducible factors of $Q\left(x_{1} t_{1 i 1}, \cdots, x_{\theta} t_{i b}\right)$ can be found by replacing in the irreducible factors of $Q\left(x_{1}{ }^{k_{k 1}}, \cdots, x_{i}{ }^{t_{k 0}}\right)$ each $x_{i}$ by $x^{a_{i}}$, which is an absurdity. Hence each set of $(\beta)$ belongs to $(\gamma)$. Similarly it can be shown that each set of $(\gamma)$ belongs to $(\beta)$. The two aggregates are therefore identical.

The sets $(\beta)$ are thus the basic sets of $Q$.

7. Suppose, next, that the polynomial $Q$ is not primary. Let $\lambda_{j}$ be the greatest common factor of the exponents of $x_{j}$ in $Q$. We have, then, that

$$
Q\left(x_{1}, \cdots, x_{s}\right)=Q^{\prime}\left(x_{1}^{\lambda_{1}}, \cdots, x_{s}^{\lambda_{s}}\right)
$$

where $Q^{\prime}\left(x_{1}, \cdots, x_{s}\right)$ is primary.

Consider the basic sets $(\beta)$ of $Q^{\prime}$. In any of these sets, say $t_{i 1}, \cdots, t_{i n}$, we replace each $t_{i j}$ by $\tau_{i j}$, where $\tau_{i j}=t_{i j} / d_{i j}$ and $d_{i j}$ is the greatest common factor of $t_{i j}$ and $\lambda_{j}$. We obtain in this way, say, $n$ sets

$$
\tau_{11}, \cdots, \tau_{18} ; \cdots ; \tau_{n 1}, \cdots, \tau_{n e} .
$$

We shall prove that the sets $\left(\beta^{\prime}\right)$ have the qualities necessary for them to be a system of basic sets of $Q$.

We verify, first, that, for any $i(i=1, \cdots, n)$, the polynomial $Q\left(x_{1}{ }^{{ }^{i i 1}}, \cdots\right.$, $\left.x_{i}{ }^{7 i}\right)$ is reducible. We have, indeed, denoting $\lambda_{i} / d_{i j}$ by $\lambda_{i j}$, that

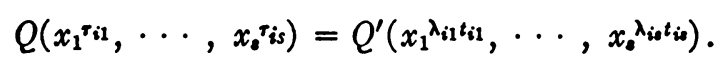

The latter polynomial is obviously reducible.

Further, if, for a given set of $t^{\prime} \mathrm{s}, Q^{(t)}$ is reducible, we find from (28) that (30) $Q^{(t)}=Q^{\prime}\left(x_{1}^{t_{1} \lambda_{1}}, \cdots, x_{s} t_{s}^{\lambda_{s}}\right)=Q_{1}\left(x_{1}^{\alpha_{1}}, \cdots, x_{s} \alpha_{s}\right) \cdots Q_{N}\left(x_{1}^{\alpha_{1}}, \cdots, x_{s}^{\alpha_{s}}\right)$.

This implies that, for every $j, t_{j} \lambda_{j}$ must be divisible by $\alpha_{j}$. Hence, if $t_{j} \lambda_{j}=\alpha_{i} \tau_{j}$ we find from (30) that

$$
Q^{\prime}\left(x^{\tau_{1}}, \cdots, x^{\tau_{s}}\right)=Q_{1}\left(x_{1}, \cdots, x_{s}\right) \cdots Q_{N}\left(x_{1}, \cdots, x_{s}\right)
$$

where all the $Q_{i}$ 's are primary. 
Consequently, the $\tau$ 's form one of the sets $(\beta)$, say the set $t_{k 1}, \cdots, t_{k e}$. For every $j$ we have, therefore,

$$
t_{i}=\frac{\alpha_{j} t_{k j}}{\lambda_{j}}=\frac{\alpha_{j} \tau_{k j}}{\lambda_{k j}} .
$$

Because $\tau_{k j}$ and $\lambda_{k j}$ are relatively prime, $\alpha_{i}$ must be divisible by $\lambda_{k j}$. Accordingly, let $\alpha_{j}=\delta_{j} \lambda_{k j}$, so that $t_{j}=\delta_{j} \tau_{k j}$.

Replacing now in (30) each $x_{i}{ }^{{ }^{8}}$ by $x_{i}$, we find that

$$
Q\left(x_{1}^{\tau_{k 1}}, \cdots, x_{s}^{\gamma_{k s}}\right)=Q_{1}\left(x_{1}^{\lambda_{k 1}}, \cdots, x_{\theta}^{\lambda_{k s}}\right) \cdots Q_{N}\left(x_{1}^{\lambda_{k 1}}, \cdots, x_{s}^{\lambda_{k s}}\right) .
$$

Hence, given a set of $t$ 's for which $Q^{(t)}$ is reducible, there exists always in $\left(\beta^{\prime}\right)$ one and only one set, say $\tau_{k 1}, \cdots, \tau_{k s}$, such that, for every $j, t_{j}=\delta_{j} \tau_{k j}$, and such that the irreducible factors of $Q^{(t)}$ are obtained by replacing in the irreducible factors of $Q\left(x_{1}^{{ }^{\tau_{k 1}}}, \cdots, x_{s}^{{ }^{\tau_{k}}}\right)$ each $x_{j}$ by $x_{j} \delta_{j}$.

We know already that there can exist no other system of sets having the same two properties.

The sets $\left(\beta^{\prime}\right)$ are, therefore, the basic sets of $Q$.

8. The results obtained in $\$ \S 6$ and 7 verify thus Theorem I, announced in the introduction. We conclude, further, on the basis of Lemma III, that Theorem II of the introduction is likewise true for a primary polynomial $Q$. If $Q$ is not primary, the upper bound for the elements of its basic sets, as we have seen, cannot exceed the corresponding bound for the basic sets of a primary polynomial whose degrees in the individual variables never exceed the degrees of $Q$ in the same variables. Hence, if $t$ is any element of any of the basic sets of $Q$, we have, a fortiori, that $t \leqq M^{2}$. Theorem II is, therefore, true for any polynomial $Q$.

9. We shall show now that the bound of Theorem II is the smallest possible bound. We shall construct a class of polynomials for which $t$ actually reaches the value $M^{2}$.

Consider the polynomial

$$
P_{0}\left(x_{1}, \cdots, x_{s}\right)=1+x_{1} x_{2} \cdots x_{s}+x_{1^{m}}^{m},
$$

where $m$ is a positive integer greater than unity.

We shall prove that $P$ is irreducible for any $m$. For, if $P_{0}=P_{1} \cdot P_{2}$, it is clear that $x_{1}$ must be present in both polynomials $P_{1}$ and $P_{2}$; otherwise, the coefficient of the last term in $P_{0}$ would be distinct from unity. This implies, however, that the polynomial

$$
R\left(x_{1}, x_{2}\right)=1+x_{1} x_{2}+x_{1}^{m}
$$

obtained from $P$ by replacing $x_{3}, x_{4}, \cdots, x_{8}$ by unity, is also reducible. If, 
therefore, $R=R_{1} \cdot R_{2}$, we conclude, for the same reason as before, that $x_{1}$ must be present in both $R_{1}$ and $R_{2}$. Because $R$ is linear in $x_{2}$, this variable can be present only in one of the factors, say $R_{1}$. Hence.

$$
1+x_{1} x_{2}+x_{1}^{m}=R_{1}\left(x_{1}, x_{2}\right) R_{2}\left(x_{1}\right) .
$$

Any root $\alpha$ of the equation $R_{2}=0$ must be distinct from zero. Replacing in (34) $x_{1}$ by $\alpha$, we find that

$$
1+x_{2} \alpha+\alpha^{m}=0,
$$

where $x_{2}$ is arbitrary, which is, of course, an absurdity. Consequently, $P_{0}$ is irreducible.

Consider the $m$ polynomials $P_{0}, P_{1}, \cdots, P_{m^{2-1}}$ obtained from $P_{0}$ by substituting $\epsilon_{1}{ }^{k_{1}} x_{1}$ for $x_{1}$, where $\epsilon_{1}$ is a primitive $m^{2}$ th root of unity and $k_{1}$ ranges over the numbers $0,1, \cdots, m^{2}-1$. Because $P_{0}$ is a primary polynomial, there will be no pair of equivalent polynomials in the set thus obtained. On the other hand, it is easy to verify that any polynomial obtained from $P_{0}$ by replacing $x_{1}$ by $\epsilon_{1}{ }^{k} x_{1} x_{1}$, where $\epsilon_{1}$ and $k_{1}$ have the same meanings as above, and by replacing, further, any other variable $x_{j}$ by $\epsilon_{j}{ }^{k} x_{j}$, where $\epsilon_{j}$ is a primitive $m$ th root of unity and $k_{j}=0, \cdots, m-1$, is identical with one of the polynomials $P_{i}$. The index $i$ is the smallest non-negative number satisfying the congruence

$$
y \equiv\left[k_{1}+m\left(k_{2}+k_{3}+\cdots+k_{8}\right)\right] \quad\left(\bmod m^{2}\right) .
$$

The product $\Pi\left(x_{1}, \cdots, x_{s}\right)$ of the $m$ polynomials $P_{i}$, therefore, remains invariant for any of the substitutions of the group $G$ described above and, consequently, is an integral rational function in $x_{1}^{m^{2}}, x_{2}^{m}, \cdots, x_{s}^{m}$.

Consider now the polynomial $\Pi_{1}\left(x_{1}, \cdots, x_{s}\right)$ obtained from $\Pi\left(x_{1}, \cdots, x_{8}\right)$ by replacing $x_{1}^{m^{2}}$ by $x_{1}$ and $x_{j}^{m}$ by $x_{j}(j=2, \cdots, s)$. The polynomial $\Pi_{1}$ is irreducible. For, if

$$
\Pi_{1}=A\left(x_{1}, \cdots, x_{s}\right) B\left(x_{1}, \cdots, x_{s}\right),
$$

then

$$
\Pi=A\left(x_{1}^{m^{2}}, x_{2}^{m}, \cdots, x_{s}^{m}\right) B\left(x_{1}^{m^{2}}, x_{2}^{m}, \cdots, x_{s}^{m}\right),
$$

a relation which is absurd, considering that the group $G$ is transitive with respect to any of the polynomials $P_{i}$ the product of which is $\Pi$.

Now, the degree of $\Pi$ in $x_{1}$ is $m^{3}$, and in any of the remaining variables is $m^{2}$. Consequently, the degrees of $\Pi_{1}$ in the same variables are all equal to $m=M$. Furthermore, replacing in $\Pi_{1}$ the variable $x_{1}$ by $x_{1}{ }^{M^{2}}$ and every other variable $x_{i}$ by $x_{i}^{M}$, we find that 


$$
\Pi_{1}\left(x_{1}^{M^{2}}, x_{2}^{M}, \cdots, x_{8}^{M}\right)=P_{0} \cdot P_{1} \cdots P_{M^{2}-1}
$$

where the $P_{i}$ 's are primary.

Hence $\Pi_{1}$ has, at least, one basic set in which one of the elements, namely $t_{1}$, actually attains the value $M^{2}$. This proves that the bound of Theorem II is the smallest possible bound.

\section{Minimal Sets}

10. From the definition of a minimal set, as given in the introduction, it follows easily that every minimal set of an irreducible polynomial consisting of more than two terms is necessarily a basic set. For its elements must be integral multiples of the corresponding elements of a particular basic set; no element, however, can be a proper multiple. Hence the minimal sets are finite in number.

11. Let $t_{1}, \cdots, t_{s}$ be the elements of a minimal set belonging to a primary irreducible polynomial $Q$. Then, in the identity

$$
Q^{(t)}=Q_{1} \cdots Q_{N}
$$

each $Q_{i}$ is primary. It is obvious, further, that not every $t_{i}$ is unity. Fixing our ideas, let us assume that $t_{1}=t_{2}=\cdots=t_{j-1}=1$ and that the remaining $t_{j}, \cdots, t_{s}$ are all greater than unity. Consider the $t_{j}$ polynomials $Q_{1}\left(x_{1}, \cdots, \epsilon_{j}{ }^{k_{j}} x_{j}, \cdots, x_{s}\right), k_{j}=0, \cdots, t_{j}-1$. We have shown above ( $\left.\$ 4\right)$ that, because $Q$ is primary, no two among these polynomials are equivalent. Denoting their product by $P$, we find that

$$
Q\left(x_{1}, \cdots, x_{j-1}, x_{j}^{t_{j}}, \cdots, x_{s}^{t_{s}}\right)=P \cdot R,
$$

where $P$ and $R$ are integral rational functions in $x_{j}{ }^{t_{j}}$. However, $R$ must be a constant; else the polynomial

$$
Q\left(x_{1}, \cdots, x_{j-1}, x_{j}, x_{j+1}^{t_{j+1}}, \cdots, x_{s}^{t_{s}}\right)
$$

would be reducible and the set $1, \cdots, 1, t_{j}, \cdots, t_{s}$ would not be a minima set. Hence $N=t_{j}$. Similarly we find that $N=t_{k}$, for $k=j, j+1, \cdots, s$. Consequently, $t_{j}=t_{j+1}=\cdots=t_{s}=t$.

12. Suppose, now, that $t=q \cdot r$, where $q$ and $r$ are positive integers and $q$ is greater than unity. The product of the $t$ polynomials $Q_{1}\left(x_{1}, \cdots\right.$, $\left.\epsilon_{j}{ }^{k_{j}} x_{j}, \cdots, x_{s}\right)$ can then be decomposed into $q$ products $P_{0}, P_{1}, \cdots, P_{q-1}$, where the factors of $P_{i}$ are the $r$ polynomials obtained by assigning to $k_{j}$ the $r$ values $i, i+q, \cdots, i+(r-1) q$. The polynomial $P_{i}$, therefore, will be invariant for any substitution which replaces $x_{j}$ by $\eta^{k} x_{j}$, where $\eta=e^{2 \pi i / r}$ and $k=0,1, \cdots, r-1$. Consequently, $P_{i}$ is a rational integral function 
in $x_{j}{ }^{r}$. We have, then, that

$$
\begin{aligned}
Q\left(x_{1}, \cdots, x_{j-1}, x_{j}{ }^{t}, \cdots, x_{s}{ }^{t}\right) & \\
\quad= & P_{0}\left(x_{1}, \cdots, x_{j}{ }^{r}, \cdots, x^{s}\right) \cdots P_{q-1}\left(x_{1}, \cdots, x_{j}{ }^{r}, \cdots, x_{s}\right)
\end{aligned}
$$

or

$$
\begin{aligned}
Q\left(x_{1}, \cdots, x_{j-1}, x_{j}^{q}, \cdots, x_{s}^{t}\right) \\
\quad=P_{0}\left(x_{1}, \cdots, x_{j}, \cdots, x_{s}\right) \cdots P_{q-1}\left(x_{1}, \cdots, x_{j}, \cdots, x_{s}\right)
\end{aligned}
$$

which is a contradiction, unless $r=1$ and $q=t$.

Consequently $t$ is a prime number. Hence, if $Q$ is primary, those elements in a minimal set which are distinct from unity are all equal to one and the same prime number.

13. We shall consider now the case when $Q$ is not primary. Referring to $\$ 7$, we shall recall that the basic sets of $Q$ are obtained from the basic sets $(\beta)$ of a certain primary polynomial by substituting for any element in each set $(\beta)$ a properly chosen factor of the element. It is clear, further, from the way these substitutions were defined, that all minimal sets of $Q$ will be found by effecting the indicated substitutions only in the minimal sets of $(\beta)$. The elements in each of the latter sets being equal either to unity or to one and the same prime number, it is obvious that the minimal sets of $Q$ will, thus, necessarily have the same structure.

14. Let $p$ be the common value of those elements in a minimal set which are distinct from unity. Because a minimal set is a basic set, and $p$ is a prime number, it follows from Theorem II that $p \leqq P$, where $P$ is the largest prime less than $M^{2}$.

The results obtained in $\$ \$ 12,13$, and 14 of this section verify thus Theorem III of the introduction.

15. We shall show now that the bound $P$, as defined in $\$ 14$, is the smallest possible bound.

Consider, indeed, the polynomial

$$
Q_{0}\left(x_{1}, \cdots, x_{8}\right)=1+x_{1}^{m-q} x_{2} \cdot x_{3} \cdots x_{8}+x_{1}^{(m-q) m-P}\left(x_{2} \cdot x_{3} \cdots x_{8}\right)^{m},
$$

where $m$ is any integer greater than unity, $P$ is the largest prime less than $m^{2}$, and $q$ is the positive integer satisfying the inequalities

$$
m^{2}-(q+1) m<P<m^{2}-q m \text {. }
$$

We prove, first, that the polynomial $Q_{0}$ is primary in all its variables. This is obvious with regard to the variables $x_{2}, x_{3}, \cdots, x_{8}$. If, on the other hand, $d$ is the greatest common divisor of the exponents in $x_{1}$, then $d$ must 
be a factor of both $m-q$ and $P$; consequently, if $d$ is distinct from unity, $P$ must be a factor of $m-q$. As $m-q<P$, we have $d=1^{*}$

We show, next, that $Q_{0}$ is irreducible. For, if $Q_{0}=Q_{1} \cdot Q_{2}$, it is clear that $x_{1}$ must be present in both $Q_{1}$ and $Q_{2}$. The polynomial

$$
R\left(x_{1}, x_{2}\right)=1+x_{1}^{m-q} x_{2}+x_{1}{ }^{(m-q)}{ }^{m-P} x_{2}{ }^{m},
$$

obtained from $Q$ by replacing each of the variables $x_{3}, \cdots, x_{8}$ by unity, is also reducible. Consider the equation $R\left(x_{1}, x_{2}\right)=0$. Treating $x_{2}$ as a function of $x_{1}$, we find, by means of Newton's polygon, that for the neighborhood of $x_{1}=0$

$$
x_{2}=\epsilon x_{1}^{-\left(m^{2}-q m-P\right) / m}+\cdots
$$

where $\epsilon$ is an $m$ th root of unity. Consequently, if $x_{1}$ describes a small circle in the complex plane about $x_{1}=0$, the $m$ branches of $x_{2}$ will be permuted in a single cycle. These $m$ branches thus hang together. Hence $R\left(x_{1}, x_{2}\right)$ cannot be reducible, which implies that $Q_{0}$ is irreducible.

Consider the $P$ polynomials $Q_{0}, Q_{1}, \cdots, Q_{P-1}$, obtained from $Q_{0}$ by substituting $\eta^{k_{1}} x_{1}$ for $x_{1}$, where $\eta$ is a $P$ th root of unity and $k=0,1, \cdots, P-1$. No two among these polynomials are equivalent. Furthermore, any polynomial obtained from $Q_{0}$ by replacing $x_{1}$ by $\eta^{k_{1}} x_{1}$ and every other variable $x_{j}$ by $\eta^{k_{i}} x_{j}$, where $k_{j}$ ranges over the same values as $k_{1}$, will be identical with one of the polynomials $Q_{i}$, say $Q_{k}$. The index $k$ is, namely, the smallest non-negative number which satisfies the congruence

$$
y(m-q) \equiv(m-q) k_{1}+k_{2}+\cdots+k_{s}
$$

We conclude, also, from the fact that $Q$ is primary in all its variables, that the same set of polynomials $Q_{i}$ will be obtained from $Q_{0}$ by means of every subgroup $g_{j}$ of substitutions which replace $x_{j}$ by $\eta^{k_{i}} x_{j}, k_{j}$ and $\eta$ having the same meanings as before, and leave all other variables unchanged.

This implies, first, that the product of the $P$ polynomials $Q_{i}$, which remains invariant for any substitution of our group, is an integral rational function $R\left(x_{1}^{P}, \cdots, x_{s}^{P}\right)$ in $x_{j}^{P}$, for every $x_{j}$. Moreover, since any of the substitutions of the subgroup $g_{j}$ simply permutes the $Q_{i}$ 's, with respect to which $g_{j}$ is transitive, we conclude that $R\left(x_{1}{ }^{P}, x_{2}{ }^{P}, \cdots, x_{j}{ }^{P}, x_{j+1}, \cdots, x_{8}\right)$, for every $j$ less than $s$, is irreducible. For, if such a polynomial were a product

then

$$
R_{1}\left(x_{1}, \cdots, x_{s}\right) \cdot R_{2}\left(x_{1}, \cdots, x_{s}\right)
$$

* According to Tchebycheff, given an integer $a$, greater than unity, there exists always a prime number between $a$ and $2 a-1$. If, therefore, $P$ is the greatest prime contained in $m^{2}$, then $P>m^{2} / 2$ and, consequently, $P>m$, considering that $m>1$. 
$R\left(x_{1}{ }^{P}, \cdots, x_{s}{ }^{P}\right)=R_{1}\left(x_{1}, \cdots, x_{j}, x_{j+1}^{P}, \cdots, x_{s}{ }^{P}\right) \cdot R_{2}\left(x_{1}, \cdots, x_{j}, x_{j+1}^{P}, \cdots, x_{s}{ }^{P}\right)$

and none of the subgroups $g_{k}(k=j+1, \cdots, s)$ could, therefore, be transitive with respect to the polynomials $Q_{i}$.

The polynomial $R\left(x_{1}, \cdots, x_{s}\right)$ obtained from $R\left(x_{1}{ }^{P}, \cdots, x_{s}{ }^{P}\right)$ by replacing each $x_{i}{ }^{P}$ by $x_{j}$, is, therefore, also irreducible. If $m_{i}$ is the degree of $Q_{0}$ in $x_{i}$, then $m_{i} P$ is the corresponding degree of $R\left(x_{1}{ }^{P}, \cdots, x_{s}{ }^{P}\right)$. Hence, the degree of $R\left(x_{1}, \cdots, x_{s}\right)$ in $x_{i}$ is $m_{i}$. Because $m_{2}=m_{3}=\cdots=m_{s}=m$ and both numbers $m-q$ and $(m-q) m-P$ are less than $m$, we see that max $\left(m_{1}, \cdots, m_{s}\right)=M=m$. The irreducible polynomial $R\left(x_{1}, \cdots, x_{s}\right)$ becomes reducible when each $x_{j}$ is replaced by $x_{j} P$. Hence the set $t_{1}=P, \cdots, t_{s}=P$ is either a minimal set of $R\left(x_{1}, \cdots, x_{s}\right)$, or is obtained from a minimal set whose elements are, then, necessarily equal either to unity or to $P$. Suppose, fixing our ideas, that its elements $\tau_{1}=\tau_{2}=\cdots=\tau_{j}=P$ and $\tau_{j+1}=\cdots$. $=\tau_{s}=1$. This implies that $R\left(x_{1}{ }^{P}, \cdots, x_{j}{ }^{P}, x_{j+1}, \cdots, x_{s}\right)$ is reducible. We have seen, however, that a polynomial of this type is always irreducible unless $j=s$.

The polynomial $R\left(x_{1}, \cdots, x_{s}\right)$ has, therefore, a minimal set all the elements of which are equal to $P$, where $P$ is the largest prime less than $M^{2}$.

\section{Polynomials of two terms}

16. We shall, for the sake of completeness, investigate the case in which the irreducible polynomial $Q$ has two terms. Changing subscripts, if necessary, we assume that

$$
Q\left(x_{1}, \cdots, x_{s}\right)=a x_{1}^{\alpha_{1}} x_{2}^{\alpha_{2}} \cdots x_{k}^{\alpha_{k}}+b x_{k+1}^{\alpha_{k+1}} \cdots x_{s}^{\alpha_{s}} .
$$

Let $t_{1}, \cdots, t_{s}$ be a set of positive integers for which $Q^{(t)}$ is reducible. We shall prove that in the identity

$$
Q^{(t)}=Q_{1} \cdots Q_{n},
$$

$Q_{1}$, and, on the basis of Lemma I*, each $Q_{i}$, contains only two terms.

Let $\lambda_{i}$ be the greatest common factor of the exponents of $x_{i}$ in $Q_{1}$. We conclude from (37) and (38) that $\alpha_{i} t_{i}$ must be divisible by $\lambda_{i}$. Hence if $\alpha_{i} t_{i}=\lambda_{i} \tau_{i}$ and if $P_{k}$ is the polynomial obtained from $Q_{k}$ by replacing each $x_{i}{ }^{\lambda_{j}}$ by $x_{i}$, we find that

$$
a x_{1}^{\tau_{1}} \cdots x_{k}^{\tau_{k}}+b x_{k+1}^{\tau_{k+1}} \cdots x_{8}^{\tau_{0}}=P_{1} \cdots P_{N},
$$

where all the $P_{i}$ 's are primary.

* In the proof of this lemma, as well as Lemma III, no restriction was made with regard to the number of terms in the irreducible polynomial $Q$. 
If each $Q_{i}$, and, consequently, each $P_{i}$, consisted of more than two terms, then, as a consequence of Lemma III, $\tau_{j} \leqq M^{2}$. Because $M$ is unity, each $\tau_{j}$ is unity, which is an absurdity. Hence, each $Q_{i}$ contains only two terms.

Since $P_{i}$ is primary and consists of only two terms, its degree in $x_{j}$ is unity. Comparing the degrees in $x_{j}$ of both sides of the identity (39) we find that $\tau_{j}=N$, for every $j$.

Consider the infinite system of sets $(\gamma)$ $t_{21}, \cdots, t_{28} ; t_{31}, \cdots, t_{3 s} ; \cdots$

where $t_{k j}=k / d_{k j}$ and $d_{k j}$ is the greatest common factor of $\alpha_{j}$ and $k$.

We easily verify that, for any set of $(\gamma)$, the corresponding $Q^{(t)}$ is reducible.

Further, if, for a given set of $t$ 's, $Q^{(t)}$ is reducible, then there exists in $(\gamma)$ one and only one set, say $t_{i 1}, \cdots, t_{i s}$, such that each $t_{j}$ is an integral multiple of $t_{i j}$ and such that if $t_{j}=\delta_{j} t_{i j}$, the irreducible factors of $Q^{(t)}$ are found by replacing in the irreducible factors of $Q\left(x_{1}{ }^{t_{i 1}}, \cdots, x_{8}{ }^{t_{i j}}\right)$ each $x_{j}$ by $x^{\delta_{i}}$.

Finally, it can be shown that there exists no other system of sets satis fyin the two condition $\mathrm{s}$ just stated.

Columbia UnIVERSITY, NEW YoRK, N. Y. 